\title{
Usefulness of Magnifying Narrow-Band Imaging Endoscopy for the Diagnosis of Gastric and Colorectal Lesions
}

\author{
Ichiro Hirata ${ }^{a}$ Yoshihito Nakagawa ${ }^{a}$ Masaaki Ohkubo ${ }^{a}$ Naohisa Yahagi ${ }^{b}$ \\ Kenshi Yaoc \\ ${ }^{a}$ Department of Gastroenterology, Fujita Health University, Toyoake, ${ }^{b}$ Cancer Center, School of Medicine, \\ Keio University, Shinjuku, and ${ }^{\complement}$ Department of Endoscopy, Fukuoka University Chikushi Hospital, Chikushino, Japan
}

\section{Key Words}

Narrow-band imaging $\cdot$ Magnifying endoscopy $\cdot$ Gastric carcinoma $\cdot$ Colorectal carcinoma $\cdot$ Colorectal adenoma . Colorectal tumor, NBI endoscopic classification .

H. pylori-associated gastritis - Intestinal metaplasia •

VS classification

\begin{abstract}
A series of studies about the potential usefulness of magnifying endoscopy with narrow-band imaging (NBI) for the diagnosis of gastric and colonic lesion is reviewed. Concerning the magnifying NBI appearances of gastric lesions, a light blue crest is a highly accurate sign of the presence of histological intestinal metaplasia. Also, the degree of irregularity of the mucosal and vascular pattern is correlated with the histological severity of Helicobacter pylori-associated chronic gastritis. According to the 'VS classification', an irregular microvascular pattern and/or an irregular microsurface pattern together with a clear demarcation line are characteristic for early gastric carcinoma, and a multicenter prospective randomized controlled trial demonstrated that magnifying endoscopy with NBI is superior to ordinary white light endoscopy for making a differential diagnosis of a small depressed lesion between carcinoma and non-carcinoma. Concerning
\end{abstract}

the magnifying NBI appearances of colonic tumor, the vague or invisible microvascular pattern is mostly observed in hyperplastic polyp. The regular meshed microvascular pattern is mostly observed in adenoma. The irregular meshed microvascular pattern is mostly observed in intramucosal or shallow submucosal-invasive carcinoma. The decreased or loose microvasucular pattern is mostly observed in deep submucosal-invasive carcinoma. Thus, magnifying NBI endoscopy is useful for the differentiation of colorectal non-adenomatous lesions from adenoma, the differentiation of adenoma from carcinoma, and the assessment of invasion depth of early colorectal carcinoma. At present, several magnifying NBI classifications for the diagnosis of early colorectal neoplasia have been proposed in Japan. Recently, the NICE classification based on NBI findings with/without magnification for colorectal tumor was established by an international group.

Copyright $\odot 2012$ S. Karger AG, Basel

\section{Introduction}

The narrow-band imaging (NBI) system is an endoscopic imaging technique for the enhanced visualization of microvascular architecture and microsurface structure

\section{KARGER}

Fax +4161306 1234 E-Mail karger@karger.ch www.karger.com
(C) 2012 S. Karger AG, Basel $0012-2823 / 12 / 0852-0074 \$ 38.00 / 0$

Accessible online at: www.karger.com/dig
Ichiro Hirata

Department of Gastroenterology, Fujita Health University, School of Medicine

1-98 Dengakugakubo, Kutsukake-cho

Toyoake city, Aichi 470-1192 (Japan)

Tel. +81 56293 9240, 2345, E-Mail ihirata@fujita-hu.ac.jp 
of the superficial part of the mucosa. Images are obtained by using narrower bands of blue and green filters, which are different from conventional red-green-blue filters [1]. Combining the NBI system and magnifying endoscopy brings a simple and clear visualization of microsurface structures and microvascular patterns of the superficial mucosa, which may be useful for precise endoscopic diagnosis, being closer to the histopathological diagnosis.

We have reviewed a series of studies investigating the potential usefulness of magnifying NBI endoscopy for the diagnosis of gastric and colonic lesion, including the contents which were discussed at the core symposia entitled 'Progression of Endoscopic Diagnosis and Treatment' of the 6th and 7th Annual Meeting of the Japan Gastroenterological Association.

\section{Stomach - Non-Neoplastic Lesions}

Although several previous studies have reported a correlation between endoscopic fine mucosal pattern seen in the gastric mucosa with magnifying endoscopy and histopathology [2-6], reports investigating a correlation between magnifying NBI appearances and pathology in non-neoplastic gastric mucosa have been relatively rare.

Uedo et al. [7] reported that observation of a light blue crest, defined as a fine, blue-white line on the crests of the epithelial surface/gyri, by using the NBI with magnifying endoscopy, is a highly accurate sign of the presence of histological intestinal metaplasia. They showed that the sensitivity and specificity of light blue crest for predicting intestinal metaplasia were 89 and $93 \%$. Bansal et al. [8] examined the feasibility of mucosal and vascular patterns seen by magnifying NBI endoscopy for predicting pathology in non-neoplastic gastric mucosa. They showed that the sensitivity and specificity of an irregular mucosal pattern with a decreased density of vessels for the diagnosis of Helicobacter pylori infection were 75 and $88 \%$, respectively, and those of the ridge/villous pattern for the diagnosis of intestinal metaplasia were 80 and $100 \%$, respectively. Tahara et al. [9] have classified the magnifying NBI appearances of non-neoplastic gastric corpus mucosa into four categories: normal and abnormal (types $1-3)$, according to the degree of irregularity of pits and microvessels (fig. 1). This classification clearly reflected the histological severity of $H$. pylori-associated chronic gastritis. Sensitivity and specificity of these abnormal types for detection of $H$. pylori positivity were 95.2 and $82.2 \%$, respectively, and those of type 3 for detection of intestinal metaplasia were 73.3 and $95.6 \%$, respectively.
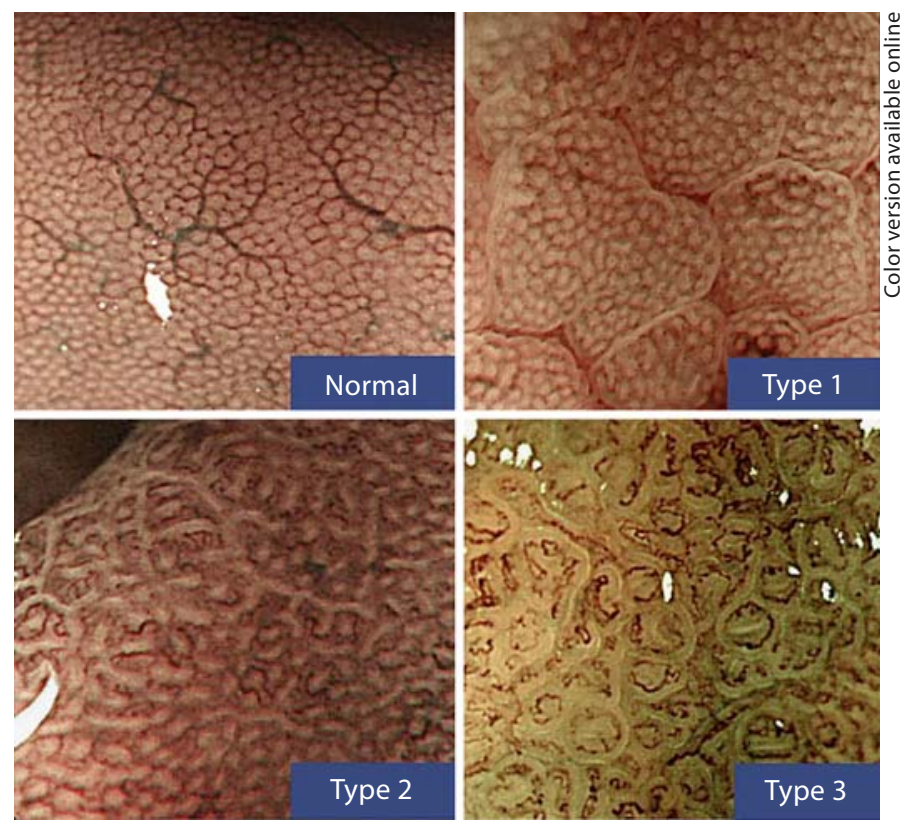

Fig. 1. Magnifying NBI endoscopic mucosal patterns of normal and $H$. pylori-related chronic gastritis in the gastric corpus. The normal pattern is characterized by a regular arrangement of small, round pits (white spots) surrounded by a subepithelial capillary network (brown rings). Type 1 is characterized by slightly enlarged, round pits with an unclear or irregular subepithelial capillary network in mild chronic gastritis mucosa. Type 2 is characterized by obviously enlarged, oval or prolonged pits with increased density of irregular vessels in moderate or severe chronic gastritis mucosa. Type 3 is characterized by well-demarcated, oval or tubulovillous pits with clearly visible coiled or wavy vessels in moderate or severe chronic gastritis mucosa with atrophy and intestinal metaplasia.

\section{Stomach - Neoplastic Lesions}

Yao et al. $[10,11]$ reported that magnifying endoscopic findings, i.e. the disappearance of a regular subepithelial capillary network pattern, presence of a demarcation line and irregular microvascular pattern, were characteristic signs for early gastric carcinoma with the histology of differentiated type (intestinal type), and the reduced microvascular pattern was for carcinoma with the histology of undifferentiated type (diffuse type). They showed that the differences in microvascular architecture, observed by magnifying endoscopy, could be useful findings for differentiating between focally reddened mucosa with gastritis and reddened flat gastric carcinoma [12, 13]. In particular, the irregular microvascular pattern was the most useful for the differential diagnosis of gastritis and carcinoma. Moreover, they showed that the 


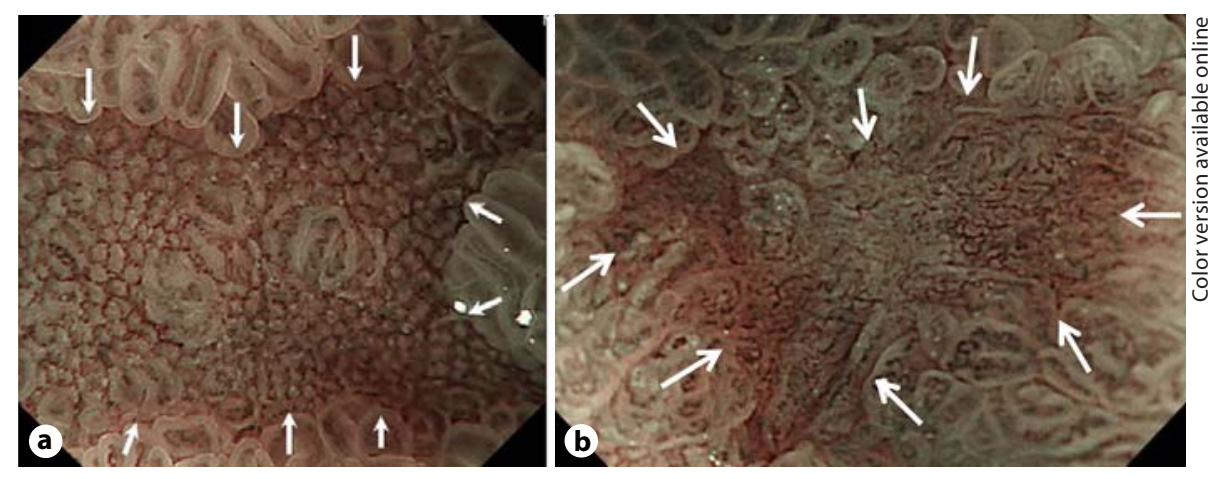

Fig. 2. a Magnifying NBI endoscopic image of focal gastritis with intestinal metaplasia in the gastric body; there is a clear demarcation line (arrows) between the background mucosa and the pathology. Within the demarcation line, there is a regular microvascular pattern (closed polygonal loop with a network) and a regular microsurface pattern (oval marginal crypt epithelium). These findings do not fulfill the criteria for carcinoma but are characteristic for focal gastritis. Only an endoscopic biopsy was performed for this lesion (quoted from [30]). b Magnifying NBI en- doscopic image of a small early gastric carcinoma (depressed type: type IIC, moderately differentiated tubular adenocarcinoma) in the gastric antrum. A clear demarcation line (arrows) was noted between the lesion and the background mucosa. Within the demarcation line, an irregular microvascular pattern (branched, tortuous, irregular open-loop and bizarre vessels) and an absent microsurface pattern are demonstrated. These findings fulfill the criteria for carcinoma. This lesion was resected by endoscopic submucosal dissection. presence of a demarcation line was useful for determining the margin of the gastric carcinoma before endoscopic submucosal dissection $[14,15]$.

Combining the NBI system and magnifying endoscopy makes clear visualization of the microanatomies which is useful for the diagnosis of early gastric carcinoma. Yao et al. [16] proposed the 'VS classification' based on a microvascular pattern and microsurface pattern, and indicated that an irregular microvascular pattern and/or an irregular microsurface pattern together with a clear demarcation line are characteristic for early gastric cancer. Recently, a multicenter prospective randomized controlled trial has been completed [17]. Also, it was demonstrated that magnifying endoscopy with NBI is superior to ordinary white light endoscopy for making a differential diagnosis of a small depressed lesion between a carcinoma and non-carcinoma (fig. 2a, b). Briefly, the sensitivity, specificity and accuracy of magnifying endoscopy with NBI versus ordinary white light endoscopy were 60.0 versus $40.0 \%(p=0.34), 90.4$ versus $64.8 \%(\mathrm{p}<0.001)$, and 90.4 versus $64.8 \%(\mathrm{p}<$ 0.001 ), respectively. This study therefore suggested that magnifying endoscopy is highly specific for making a diagnosis of carcinoma and that it could contribute to reducing the number of biopsies which are taken from benign lesions.

Nonaka et al. [18] classified 93 lesions, mostly flat elevated lesions, into five types (types I-V) based on the mucosal microstructure and microvessels, namely type I: clear mucosal microstructure and unclear microvessel image; type II: clear mucosal microstructure and clear microvessel image; type III: clear mucosal microstructure and abnormal microvessel image; type IV: slightly obscured mucosal microstructure and abnormal microvessel image, and type V: markedly obscured mucosal microstructure and abnormal microvessel image. They also mentioned the feasibility of endoscopic differentiation of gastric adenoma from well-differentiated adenocarcinoma according to these types.

\section{Colon - Neoplastic Lesions}

Magnified colonoscopy and the development of pit pattern diagnosis are useful for the diagnosis of colorectal polypoid lesions and the assessment of invasion depth of early colorectal carcinoma. Recently, the NBI system has been developed besides these diagnostics. NBI is useful for the differentiation of colorectal non-adenomatous lesions from adenoma, and for the differentiation of adenoma from carcinoma (fig. 3a, b). Moreover, it is helpful for the assessment of invasion depth of early colorectal carcinoma without dye spraying [19-21]. In addition, it is used for cancer surveillance in inflammatory bowel disease [22] or hereditary non-polyposis colorectal cancer in which the proportion of flat adenomas detected was sig- 
nificantly higher using NBI endoscopy than using conventional white-light endoscopy [23].

At present, several magnifying NBI classifications for the diagnosis of colorectal tumor have been proposed in Japan. We introduced and explained the leading magnifying NBI classifications in Japan as well as the NBI International Colorectal Endoscopic (NICE) classification.

\section{Sano Classification [24-26]}

The Sano classification is based on the evaluation of the microvascular architecture on the surface of lesions. The microvascular architecture (capillary pattern) was classified into I, II, IIIA, or IIIB. The capillary pattern considered the arrangement of the meshed capillary surrounding the mucosal glands. Type I is characterized by meshed capillary vessels being visually unidentifiable. Type I is mostly observed in hyperplastic polyps. Type II which is mostly observed in adenoma is characterized by meshed capillary vessels which are clearly visualized and surround mucosal glands. Type III mostly observed in carcinomas is characterized by meshed capillary vessels showing a blind ending, branching and being irregularly curtailed. Type III is divided into two subtypes: type IIIA characterized by high microvessel density with lack of uniformity, and type IIIB characterized by the presence of the area showing nearly avascular or loose microvascular. Type IIIB is observed in deep submucosal (SM)invasive carcinomas.

\section{Hiroshima Classification $[19,21]$}

The Hiroshima classification is based on the evaluation of both microvascular architecture and 'pit-like pattern' on the surface of the lesions. The 'pit-like pattern' identified the white part on the surface, which is similar to the pit structure. This classification consists of five types: type A, B, C1, C2, and C3. Type A observed mostly in hyperplastic polyp is characterized by vague or invisible microvessel intensity. Type $B$ is characterized by a clear and regular pit-like pattern (surface pattern) with the increased microvessel intensity around the pits or regular meshed microvessel network pattern. Type B is mostly observed in adenoma or cancer in adenoma. Type $\mathrm{C} 1$ is characterized by irregular surface pattern with the increased microvessel intensity around the pits and homogeneous vascular thickness and distribution. Type $\mathrm{C} 1$ is observed in intramucosal carcinoma and shallow SMinvasive carcinoma. Type $\mathrm{C} 2$ is characterized by more irregularity of the surface pattern and more increased microvessel intensity than type $\mathrm{C} 1$ shows, and heterogeneous thickness and distribution of vessels. Type C2 is

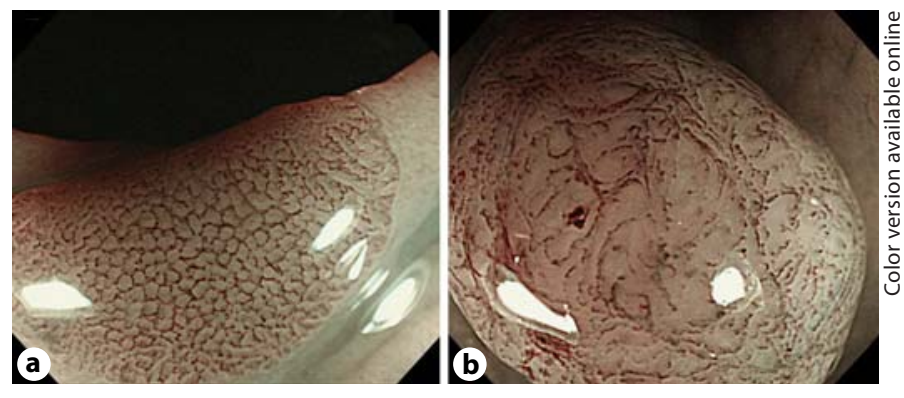

Fig. 3. a Magnifying NBI endoscopic image of colonic adenoma (superficial elevated type: type IIA, tubular adenoma). The regular meshed arrangement of the thick brown microvessels surrounding the polygonal white structures (surface pattern or pitlike pattern, which represents the pits and the epithelium of the crypt opening) is demonstrated. These findings are characteristic of adenoma corresponding to Sano type II, Hiroshima type B, Showa network pattern, Jikei type 2, and NICE type 2. This lesion was resected by endoscopic mucosal resection. b Magnifying NBI endoscopic image of colonic carcinoma (protruded type: type IS, tubular adenocarcinoma with deep SM invasion). Sparse and partially absent microvessels with an unclear surface pattern are demonstrated. These findings are characteristic of deep SM-invasive adenocarcinoma corresponding to Sano type IIIB, Hiroshima type C3, Showa sparse pattern, Jikei type 4, and NICE type 3. Surgical operation was performed for this lesion.

observed in both intramucosal carcinoma and SM-invasive carcinoma. Approximately $60 \%$ of type C2 tumors are deep SM-invasive carcinomas. Type C3 is characterized by an unclear surface pattern with an avascular area or scattered microvessel fragments area. Type C3 is observed in deep SM-invasive carcinomas.

\section{Showa Classification [27]}

The Showa classification is based on the evaluation of the microvasucular architecture. This classification does not use the symbol such as type I or type A for categorization. The evaluated data are categorized according to the findings of vessel changes and classified into six categories: normal, faint, network, dense, irregular, and sparse pattern. The faint pattern is characterized by which microvessel surrounding the gland is difficult to be visually identified. The faint pattern is mostly observed in hyperplastic polyps. The network pattern is characterized by regular meshed microvessel surrounding the gland and is observed in tubular adenoma and intramucosal carcinoma, mostly in tubular adenoma. The dense pattern is characterized by thick and dense microvessel surrounding the gland and is observed in villous/tubulovillous adenoma and intramucosal carcinoma, mostly in villous/ 
tubulovillous adenoma. The irregular pattern is characterized by microvessel with irregular large caliber, high tortuosity, and interruption of microvessel network. The sparse pattern is characterized by or scattered microvessel fragments avascular due to high deterioration of microvessels. The irregular and sparse pattern is observed in deep SM-invasive carcinomas.

\section{Jikei Classification [28]}

The Jikei classification is based on the evaluation of the degree of the microvessel caliber dilatation and partial evaluation of 'pit-like pattern'. This classification consists of five types: $1,2,3 \mathrm{~V}, 3 \mathrm{I}$, and 4 . Type 1 is characterized by no recognition of the microvessel pattern. Type 2 is characterized by the slightly increased vascular diameter. Type 3 is characterized by the remarkably increased vascular diameter. Type 3 is subclassified into $3 \mathrm{~V}$ showing the regular microvessel pattern with a villous pit-like pattern and 3I showing an irregular microvessel pattern without a pit-like pattern. Type 4 is characterized by the sparse distribution of microvessels.

\section{NICE Classification [19, 21]}

The NICE classification was established by an international cooperative group (Colon Tumor NBI Interest Group - CTNIG) including Japanese, USA, French and UK endoscopists [19]. The NICE classification is based on the evaluation of the following three NBI characteristics in colorectal tumor: color, vessels, and surface pattern, both with or without using a magnifying endoscope. It consists of three types: types 1-3. Type 1 is characterized by the color being the same or lighter than the background, no or isolated lacy vessels and the surface pattern is dark or white spots of uniform size, or homogeneous absence of pattern. Type 1 is considered an index for hyperplastic lesions. Type 2 is characterized by the color being browner relative to the background, thick brown vessels surrounding white structures and the surface pattern being oval, tubular or branched white structures sur- rounded by brown vessels. Type 2 is considered an index for adenoma or mucosal/scanty SM-invasive carcinoma. Type 3 is characterized by the color being brown to dark brown relative to the background; sometimes a patchy whiter area, markedly distorted or missing vessels, and areas showing distortion or absence of surface pattern. Type 3 is considered an index for deep SM-invasive carcinoma.

Currently, there are no comparative data among all of these classifications concerning the diagnostic accuracy for the malignancy of colorectal tumor and invasion depth of early colorectal carcinoma. It is therefore difficult to objectively comment on the advantage of each of these classifications. Although it was a retrospective study, the paper reported that magnifying NBI endoscopy is comparable to magnifying chromoendoscopy on evaluating invasion depth of early colorectal carcinoma, but there was greater interobserver variability in the diagnosis using NBI [29].

Unification of terminology and classification of NBI endoscopy for colorectal tumor need to be established and a prospective randomized controlled trial needs to be performed using such a classification in the near future.

To summarize, magnifying NBI endoscopy is expected to become an excellent diagnostic method for gastric and colorectal lesions by clearly demonstrating the mucosal surface structure and microvascular pattern. However, more evidence is needed in order to determine whether magnifying NBI endoscopy is comparable or superior to magnifying chromoendoscopy on making a diagnosis for gastrointestinal lesions, especially for colonic lesions.

\section{Disclosure Statement}

The authors declare that no financial conflict or conflict of interest exists in relation to the contents of this article.

\section{References}

1 Gono K, Obi T, Yamaguchi M, Ohyama N, Machida H, Sano Y, Yoshida S, Hamamoto Y, Endo T: Appearance of enhanced tissue features in narrow-band endoscopic imaging. J Biomed Opt 2004;9:568-577.

-2 Sakaki N, Iida Y, Okazaki Y, Kawamura S, Takemoto T: Magnifying endoscopic observation of the gastric mucosa, particularly in patients with atrophic gastritis. Endoscopy 1978;10:269-274.
-3 Yagi K, Nakamura A, Sekine A: Characteristic endoscopic and magnified endoscopic findings in the normal stomach without $\mathrm{He}$ licobacter pylori infection. J Gastroenterol Hepatol 2002;17:39-45.

-4 Yagi K, Nakamura A, Sekine A: Comparison between magnifying endoscopy and histological, culture and urease test findings from the gastric mucosa of the corpus. Endoscopy 2002;34:376-381. 
5 Nakagawa S, Kato M, Shimizu Y, Nakagawa M, Yamamoto J, Luis PA, Kodaira J, Kawarasaki M, Takeda H, Sugiyama T, Asaka M: Relationship between histopathologic gastritis and mucosal microvascularity: observations with magnifying endoscopy. Gastrointest Endosc 2003;58:71-75.

-6 Anagnostopoulos GK, Yao K, Kaye P, Fogden E, Fortun P, Shonde A, Foley S, Sunil S, Atherton JJ, Hawkey C, Ragunath K: Highresolution magnification endoscopy can reliably identify normal gastric mucosa, Helicobacter pylori-associated gastritis, and gastric atrophy. Endoscopy 2007;39:202-207.

$>7$ Uedo N, Ishihara R, Iishi H, Yamamoto S, Yamamoto S, Yamada T, Imanaka K, Takeuchi Y, Higashino K, Ishiguro S, Tatsuta M: A new method of diagnosing gastric intestinal metaplasia: narrow-band imaging with magnifying endoscopy. Endoscopy 2006;38:819-824.

$>8$ Bansal A, Ulusarac O, Mathur S, Sharma P: Correlation between narrow band imaging and non-neoplastic gastric pathology: a pilot feasibility trial. Gastrointest Endosc 2008; 67:210-216

-9 Tahara T, Shibata T, Nakamura M, Yoshioka D, Okubo M, Arisawa T, Hirata I: Gastric mucosal pattern by using magnifying narrow-band imaging endoscopy clearly distinguishes histological and serological severity of chronic gastritis. Gastrointest Endosc 2009;70:246-253.

10 Yao K, Oishi T: Microgastroscopic findings of mucosal microvascular architecture as visualized by magnifying endoscopy. Dig Endosc 2001;13:S27-S33.

- 11 Yao K, Oishi T, Matsui T, Yao T, Iwashita A: Novel magnified endoscopic findings of microvascular architecture in intramucosal gastric cancer. Gastrointest Endsc 2002;56: 279-284.

-12 Yao K, Iwashita A, Kikuchi Y, Yao T, Matsui T, Tanabe H, Nagahama T, Sou S: Novel zoom endoscopy technique for visualizing the microvascular architecture in gastric mucosa. Clin Gastroenterol Hepatol 2005; 3(suppl 1):S23-S26.

- 13 Yao K, Iwashita A, Tanabe H, Nagahama T, Matsui T, Ueki T, Sou S, Kikuchi Y, Yorioka M: Novel zoom endoscopy technique for diagnosis of small flat gastric cancer: a prospective, blind study. Clin Gastroenterol Hepatol 2007;5:869-878.
14 Yao K, Yao T, Iwashita A: Determining the horizontal extent of early gastric carcinoma: two modern techniques based on differences in the mucosal microvascular architecture and density between carcinomatous and non-carcinomatous mucosa. Dig Endosc 2002;14:S83-S87.

15 Yao K, Kikuchi Y, Tanabe H: Novel zoom endoscopy technique for visualizing the microvascular architecture of early gastric cancer enables the precise margin of the cancer to be determined thereby allowing successful resection by the endoscopic submucosal dissection method. Endoscopy 2004;36:A6.

16 Yao K, Anagnostopoulos GK, Ragunath K: Magnifying endoscopy for diagnosis and delineating early gastric cancer. Endoscopy 2009;41:462-467.

17 Uedo N, Ezoe Y, Muto M, Takeuchi Y, Doyama H, Kaneko Y, Oda I, Yao K, Kaneko K, Yano T, Kawahara Y, Yokoi C, Sugiura Y, Ishikawa H, Saito Y: Differential diagnosis of small gastric depressed lesions by magnifying narrow band imaging: a multicenter prospective randomized controlled trial. Gastrointest Endosc 2011;73(suppl):AB146.

18 Nonaka K, Arai S, Ban S, Kitada H, Namoto M, Nagata K, Ochiai Y, Togawa O, Nakao M, Nishimura M, Ishikawa K, Sasaki Y, Kita H: Prospective study of the evaluation of the usefulness of tumor typing by narrow band imaging for the differential diagnosis of gastric adenoma and well-differentiated adenocarcinoma. Dig Endosc 2011;23:146-152.

19 Oba S, Tanaka S, Sano Y, Oka S, Chayama K: Current status of narrow-band imaging magnifying colonoscopy for colorectal neoplasia in Japan. Digestion 2011;83:167-172.

20 Fukuzawa M, Saito Y, Matsuda T, Uraoka T, Itoi T, Moriyasu F: Effectiveness of narrowband imaging magnification for invasion depth in early colorectal cancer. World J Gastroenterol 2010;16:1727-1734.
21 Tanaka S, Sano Y: Aim to unify the narrowband imaging magnifying classification for colorectal tumors: current status in Japan from a summary of the consensus symposium in the 79th Annual Meeting of the Japan Gastroenterological Endoscopy Society. Dig Endosc 2011;23(suppl 1):131-139.

22 Ahmed T, Monti J, Lashner B: Random versus targeted biopsies for colorectal cancer surveillance in inflammatory bowel disease. Gastroenterol Hepatol 2010;6:438-442.

23 Lee MM, Enns R: Narrow band imaging for the detection of neoplastic lesions of the colon. Can J Gastroenterol 2009;23:15-18.

24 Sano Y: Narrow band imaging (English abstract) Clin Gastroenterol 2009;24:47-52.

$>25$ Ikematsu H, Matsuda T, Emura F, Saito Y, Uraoka T, Fu KI, Kaneko K, Ochiai A, Fujimori T, Sano Y: Efficacy of capillary pattern type IIIA/IIIB by magnifying narrow band imaging for estimating depth of invasion of early colorectal neoplasms. BMC Gastroenterol 2010;27:10:33

26 Uraoka T, Saito Y, Ikematsu H, Yamamoto K, Sano Y: Sano's capillary pattern classification for narrow-band imaging of early colorectal lesions. Dig Endosc 2011;23(suppl 1):112-115

27 Wada Y, Kashida H, Kudo S, Misawa M, Ikehara N, Hamatani S: Diagnostic accuracy of pit pattern and vascular pattern analyses in colorectal lesions. Dig Endosc 2010;22:192199.

28 Saito S, Nikami T, Aihara H, Arakawa H, Tajiri H, Ikegami M: Observation of capillary pattern might be helpful finding to diagnose of depth of invasion in early colon cancer by using NBI systems (English abstract). Intestine 2009;13:209-213.

29 Sakamoto T, Saito Y, Nakajima T, Matsuda T: Comparison of magnifying chromoendoscopy and narrow-band imaging in estimation of early colorectal cancer invasion depth: a pilot study. Dig Endosc 2011;23:118123.

-30 Yao K, Takaki Y, Nagahama T, et al: Clinical applications of optical equipment-based image enhanced endoscopy within the upper gastrointestinal tract (in Japanese). J Jpn Soc Gastroenterol 2011;108:887-898. 